\title{
A long range dependent model with nonlinear innovations for simulating daily river flows
}

\author{
P. Elek and L. Márkus \\ Department of Probability Theory and Statistics, Eötvös Loránd University, Budapest, Hungary \\ Received: 30 September 2003 - Revised: 11 February 2004 - Accepted: 5 March 2004 - Published: 16 April 2004 \\ Part of Special Issue "Multidisciplinary approaches in natural hazards"
}

\begin{abstract}
We present the analysis aimed at the estimation of flood risks of Tisza River in Hungary on the basis of daily river discharge data registered in the last 100 years. The deseasonalised series has skewed and leptokurtic distribution and various methods suggest that it possesses substantial long memory. This motivates the attempt to fit a fractional ARIMA model with non-Gaussian innovations as a first step. Synthetic streamflow series can then be generated from the bootstrapped innovations. However, there remains a significant difference between the empirical and the synthetic density functions as well as the quantiles. This brings attention to the fact that the innovations are not independent, both their squares and absolute values are autocorrelated. Furthermore, the innovations display non-seasonal periods of high and low variances. This behaviour is characteristic to generalised autoregressive conditional heteroscedastic (GARCH) models. However, when innovations are simulated as GARCH processes, the quantiles and extremes of the discharge series are heavily overestimated. Therefore we suggest to fit a smooth transition GARCH-process to the innovations. In a standard GARCH model the dependence of the variance on the lagged innovation is quadratic whereas in our proposed model it is a bounded function. While preserving long memory and eliminating the correlation from both the generating noise and from its square, the new model is superior to the previously mentioned ones in approximating the probability density, the high quantiles and the extremal behaviour of the empirical river flows.
\end{abstract}

\section{Introduction}

River Tisza - the second largest in Hungary - has a long history of damaging floods even after the river was controlled in the nineteenth century. The record water levels in years 2000 and 2001 drew again the attention to the question how high

Correspondence to: P. Elek

(elekpeti@cs.elte.hu) dams should be built in order to prevent a huge flood catastrophe in the Great Hungarian Plain. So the estimation of high quantiles of the discharge series has again become crucial. Beyond that, studying the behaviour of the whole process is also important because the river provides irrigation water for a large agricultural area in the Hungarian Plain. In the past, the extremal analysis and the conventional time series analysis for River Tisza were usually carried out in separate studies. This paper attempts to incorporate both approaches: the aim is to find a time series model which describes both the regular and the extremal behaviour of the process.

The data we have at our disposal consists of daily water discharges from 1901 to 2000 at six monitoring stations along the river (Tivadar, Vásárosnamény, Záhony, Polgár, Szolnok and Szeged). To obtain a visual impression of the data, we display on Fig. 1 the discharge series registered at Vásárosnamény station. It turns out that all six series exhibit a substantial linear and seasonal trend both in their mean and their variance. We used a classical approach to tackle this problem: first a linear and a periodic trend component was subtracted from the data at each station and then these meancorrected series were standardised by a periodic factor to make the variance roughly constant over time. The periodic components were estimated using the loess smoother procedure proposed by Cleveland et al. (1990). The drawback of this procedure is that the standardised series - although stationary in mean and variance - still exhibit seasonal change in their probability density functions. We will see that this problem can partly be resolved during simulations.

All six standardised series exhibit substantial long range dependence as evidenced by various nonparametric estimators (autocorrelation-based method, rescaled-range statistics, aggregate variance method and Geweke-Porter-Hudak estimator etc.). A usual way to model such series is to fit a fractional ARIMA process which can then be used to simulate synthetic streamflow series in a straightforward way. (The detection of long range dependence (LRD) or long memory in certain hydrologic time series dates back to the early works of Hurst (1951) and since then a plenty of articles have dealt 


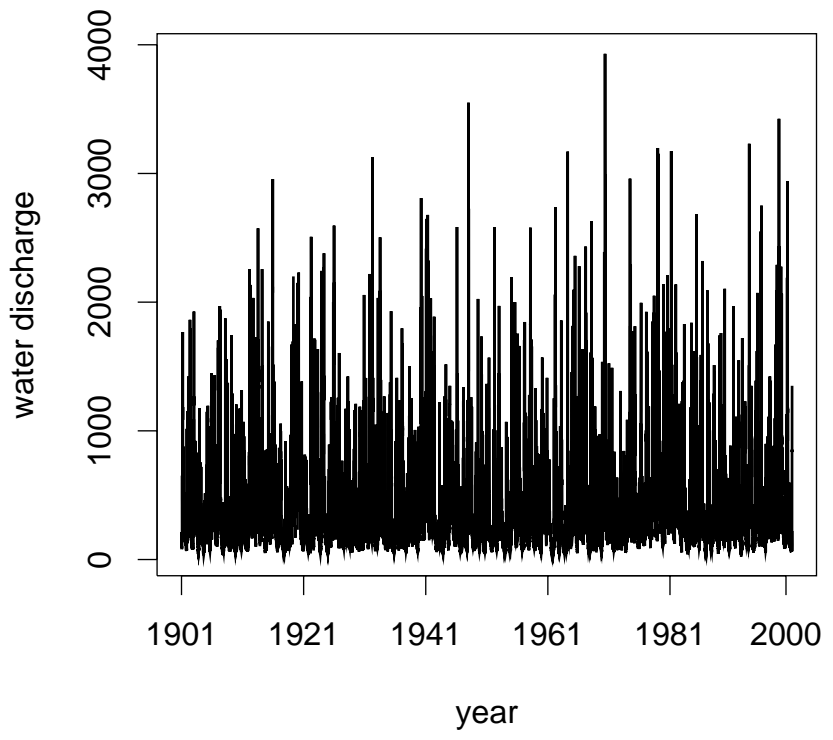

Fig. 1. Daily discharge series at Vásárosnamény $\left(\mathrm{m}^{3} / \mathrm{s}\right)$, 1901-2000.

with this phenomenon. For a recent example see Montanari et al. (1997) and for a monograph see Beran (1994).)

Although this simulation method gives back the autocorrelation-structure of the water discharge series of River Tisza accurately, it is not suitable for flood risk estimation because the high quantiles of the synthetic series remain well below the empirical ones. The reason is that the innovations of the fractional ARIMA model - which are non-Gaussian - are uncorrelated but not independent. This phenomenon is consistent with the fact that hydrologic time series are highly nonlinear, so a linear model may not give back the whole complexity of the process.

Innovations can be regarded as shocks to the linear system. They are uncorrelated but their squares and absolute values are autocorrelated and, additionally, they exhibit nonseasonal periods of high and low variances with high variance generally occuring during unusual weather events. These properties suggest modelling the innovations with a variant of the standard GARCH process. (GARCH processes were introduced by Bollerslev in 1986 and since then have been widely used especially in financial mathematics.) However, as hydrologic time series are less heavy-tailed than financial ones, the models should differ as well. In a standard GARCH process the dependence of the variance on the lagged innovations is quadratic, whereas in our model it is a bounded function.

Our approach for simulation is thus the following: we fit a GARCH-type model to the innovation series, estimate the GARCH residuals and then use a resampling procedure to simulate them. In doing so, we take into consideration the seasonally changing shape of their probability density as well. We then apply the GARCH model to get back the innovations of the linear system. Finally we drive the fractional ARIMA filter with the innovations and use the seasonal com-
Table 1. Results of fractional ARIMA fit

\begin{tabular}{lcccc}
\hline Monitoring station & $\mathrm{p}$ & $\mathrm{q}$ & Hurst-parameter & $\mathrm{p}$-value \\
\hline Tivadar & 1 & 1 & $0.748(0.022)$ & 0.893 \\
Vásárosnamény & 2 & 1 & $0.821(0.014)$ & 0.759 \\
Záhony & 2 & 1 & $0.804(0.018)$ & 0.738 \\
Polgár & 2 & 1 & $0.794(0.026)$ & 0.451 \\
Szolnok & 2 & 3 & $0.752(0.034)$ & 0.051 \\
Szeged & 2 & 2 & $0.844(0.030)$ & 0.057 \\
\hline
\end{tabular}

ponents to obtain synthetic streamflow series. This model, by incorporating time-varying nonseasonal variance, estimates the probability density and high quantiles of the observed series much better than a linear model. It brings us closer to understanding the nonlinear nature of hydrologic time series.

\section{Fitting a fractional ARIMA model}

In view of the long memory property we first fitted the series with a fractional ARIMA (FARIMA) process $X_{t}$ satisfying

$\Phi_{p}(B)(1-B)^{d} X_{t}=\Psi_{q}(B) \varepsilon_{t}$.

Here $B$ is the backward shift operator, $d$ is the order of fractional differencing, $\varepsilon_{t}$ is the uncorrelated and zero-mean innovation (noise) sequence with variance $\sigma_{\varepsilon}^{2}$, and, in the notations of ARMA-methodology,

$$
\begin{aligned}
& \Phi_{p}(B)=1-\sum_{j=1}^{p} \phi_{j} B^{j} \\
& \Psi_{q}(B)=1+\sum_{j=1}^{q} \psi_{j} B^{j} .
\end{aligned}
$$

In cases of our interest $d$ lies within 0 and 0.5. The Hurstparameter is then $H=d+1 / 2$.

A FARIMA(p, $\mathrm{d}, \mathrm{q})$ model has $p+q+2$ parameters: $p+q$ for the ARMA-coefficients, one for the fractional differencing parameter (these together are called the structural parameters) and one for the variance of the innovation process. In the following, we denote the structural parameters by $\boldsymbol{\theta}$. The parameters can be estimated by various methods, including exact normal-based maximum likelihood procedure or the Whittle-estimator. The latter, which we used, is essentially an approximation of the log-likelihood function in the spectral domain. According to Giraitis and Surgailis (1990), the Whittle-estimator is consistent and asymptotically normal for linear processes with finite fourth moment. However, these properties cease to hold for certain nonlinear processes, see e.g. Giraitis and Taqqu (1999).

Goodness of fit of a FARIMA model can be tested with a method based on the sum of squares of all autocorrelations of the estimated innovation process (Beran, 1992). The test statistic (under the condition of normal generating noise) is 


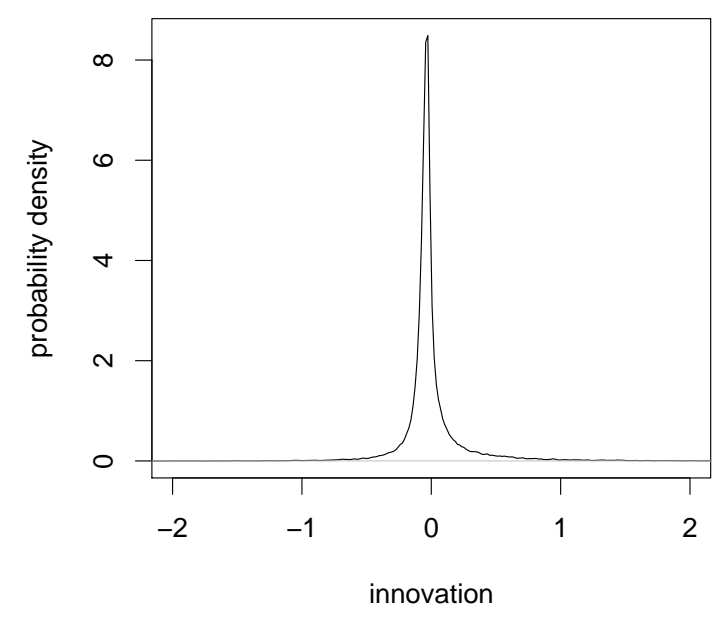

Fig. 2. Probability density of innovations at Vásárosnamény.

asymptotically normal and thus a p-value can easily be calculated. For model identification (i.e. choosing the appropriate value of $p$ and $q$ ) we used a trial and error procedure and then evaluated the different models by the $\mathrm{p}$-value of the goodness of fit statistics and by the significance level of the parameters. Table 1 shows the most important features of the finally fitted models at all sites. As the values with the standard errors (in parentheses) show, the Hurst-parameters significantly exceed 0.5 , implying long memory in all cases.

According to the p-values in the table, all six models are acceptable at 5\% significance level. (The lower p-values at Szolnok and Szeged are most probably resulted from the fact that reservoirs are operated just upstream of these stations.) The good fit is also expressed by the uncorrelatedness of innovations at all stations. In other words, the linear model fully describes the linear dependence structure, that is, the autocovariances of the series. The innovations were obtained using a finite approximation of the inverse of the estimated FARIMA filter:

$\tilde{\varepsilon}_{t}=\Psi_{q}(B)^{-1} \Phi_{p}(B)(1-B)^{d} X_{t} \approx \sum_{j=0}^{200} b_{j} X_{t-j}$.

\section{Simulations from the fractional ARIMA model}

Nevertheless, from the natural hazards (flood) perspective, the main question is the linear model's goodness of fit in terms of distribution and high quantiles. To examine that, we need to simulate water discharge series from the model. If the independence of innovations is assumed, the simulation can be carried out in the following straightforward way.

First, synthetic $\epsilon_{t}$ innovations are generated. As their distribution is highly non-Gaussian (see Fig. 2), a seasonal bootstrap procedure is applied: a synthetic innovation in month $\mathrm{A}$ is randomly selected from all observed innovations in the same month of a possibly different year. This method (used e.g. by Montanari et al., 1997) has the advantage of not making any artificial distributional assumptions, but it has some
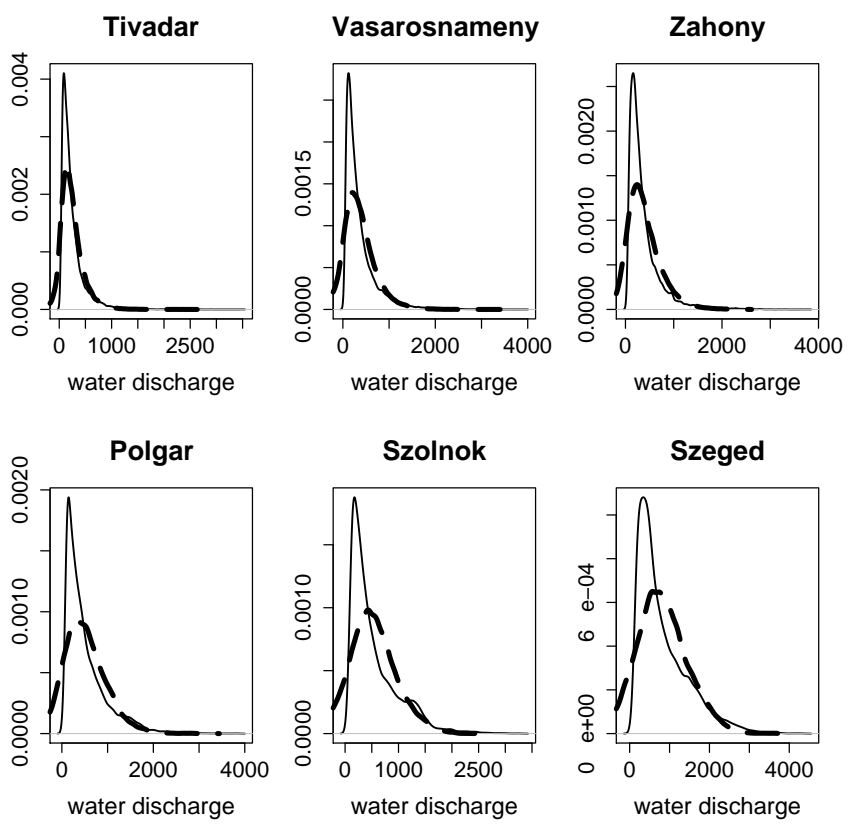

Fig. 3. Probability density of observed (continuous line) and of linearly simulated (dashed line) discharge series (in $\mathrm{m}^{3} / \mathrm{s}$ ) at different stations.

serious drawbacks, being very sensitive to extreme observations.

In the second step, $X_{t}$ is obtained from $\epsilon_{t}$ using the moving average representation of fractional ARIMA processes. In practise, we used innovations up to lag 200:

$X_{t}=(1-B)^{-d} \Phi_{p}(B)^{-1} \Psi_{q}(B) \varepsilon_{t} \approx \sum_{j=0}^{200} a_{j} \varepsilon_{t-j}$

Finally, giving back the seasonal components (both in mean and variance) to $X_{t}$, synthetic water discharge series are generated.

According to Montanari et al. (1997), this simulation method provides a reasonably good approximation of the sample density of daily inflows to Lake Maggiore in Italy. However, in the case of River Tisza, we arrive at different conclusions. Figure 3 shows the probability densities of the observed and simulated water discharge series at all monitoring stations. The goodness of fit is not appropriate at any stations, becoming even worse while getting downstream. Figure 4 shows the same: discharge quantiles (displayed at 90, 95, 99, 99.5, 99.9, 99.95\% levels) and the 100-year maximum are seriously underestimated by the linear model. For instance, when 100-year long synthetic series are generated twenty times, the median value of their maxima is smaller than the discharge 100-year maxima by $20-25 \%$ (depending on which site is examined). Thus, we can conclude that as far as sample densities and high quantiles are concerned, the linear model is not appropriate for modelling water discharge series of River Tisza. 

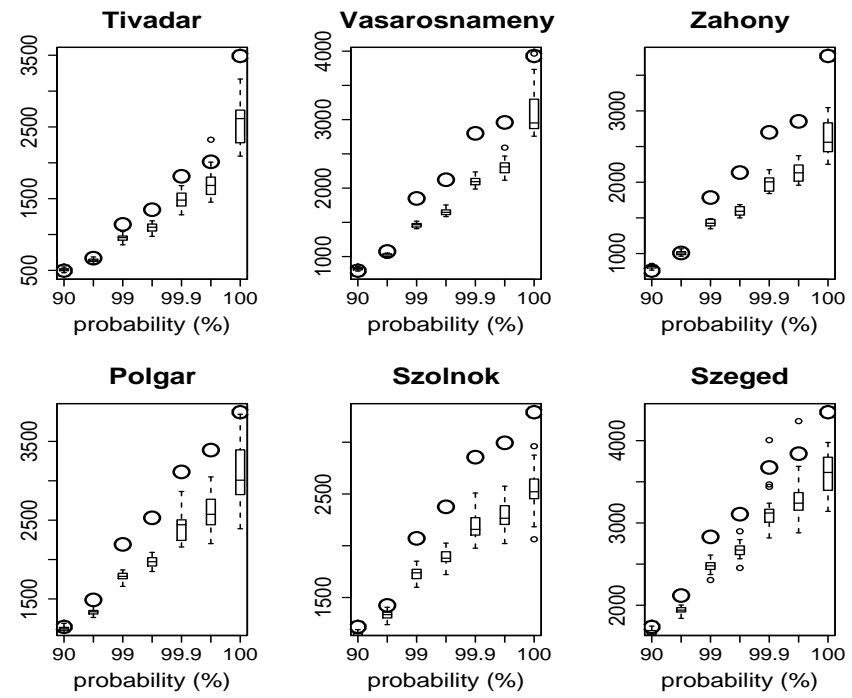

Fig. 4. Boxplots of quantiles, displayed at 90, 95, 99, 99.5, 99.9, $99.95 \%$ levels, and of sample maxima of 20 simulated series at each site, compared to the observed values (circles). Boxplots contain the median value, the quartiles and the extreme observations.
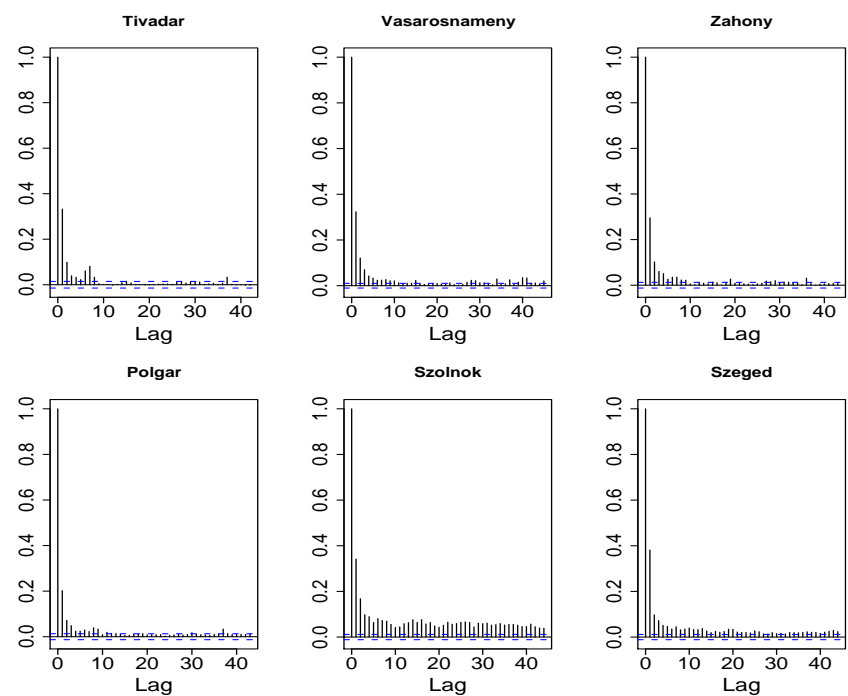

Fig. 5. Autocorrelation function of squared innovations at different sites.

\section{Incorporating the structure of innovations}

The above described model has one point at which it fails: it does not incorporate the nonlinear dependence structure of the innovations. As evidenced by Figs. 5 and 6, the innovations are dependent through their squares and their absolute values. (The autocorrelatedness of the squared and the absolute valued innovation series, respectively, can be proven by a Ljung-Box-test at all reasonable significance levels.) Generating innovations by bootstrap procedure eliminates interdependence, thus important information on higher order dependence is lost.
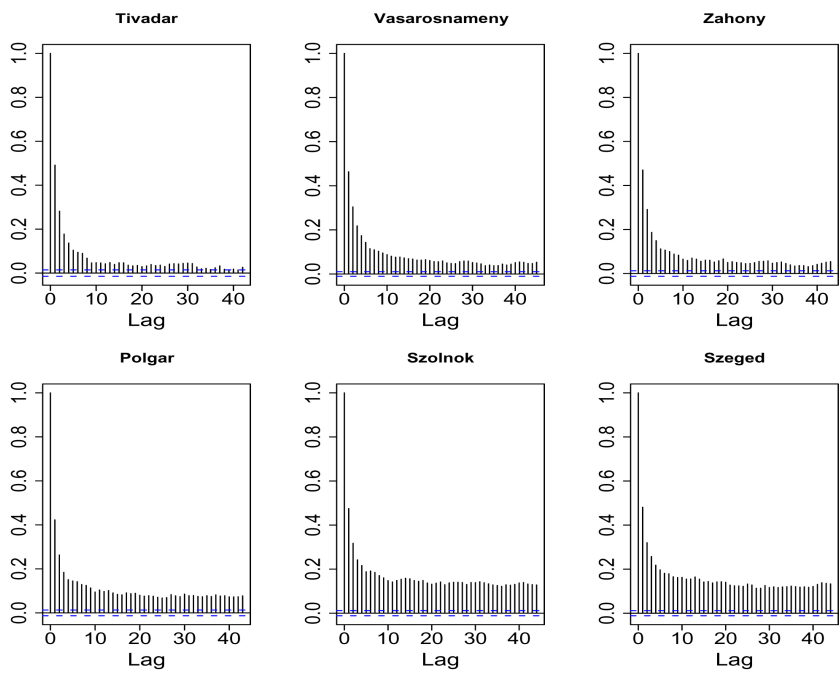

Fig. 6. Autocorrelation function of absolute values of innovations at different sites.

When taking a closer look at the structure of the innovations, one can observe that it is a conditionally heteroscedastic process: periods of high variance are followed irregularly by less variable periods. This heteroscedasticity is beyond seasonal variation because the latter was eliminated in the standardization procedure. The phenomenon can be called "variance clustering" because innovations with high absolute values (i.e. with high conditional variance) tend to appear in clusters. The clustering also explaines why the squared innovation series is autocorrelated.

To demonstrate the clustering effect, we can estimate the variance at time $t$ by the method of moving averages, i.e. by averaging the squared values of innovations from time $t-M / 2$ until time $t+M / 2$ (here we use the fact that the mean is close to zero):

$\operatorname{Var}(\varepsilon)_{t}=\frac{1}{M+1} \sum_{j=-M / 2}^{M / 2} \varepsilon_{t-j}^{2}$.

When we take e.g. $M=10$ for a 10 years long subseries, as on Fig. 7, the estimated variance process of the original innovation series differs substantially in quantiles and in maximum from that of an independent series obtained by reshuffling. For instance, at Vásárosnamény, the maximum of the former exceeds the maximum of the latter by $38 \%$. This underscores the importance of modelling the clustering effect.

\section{Fitting a FARIMA-GARCH model}

Series exhibiting variance clustering and other related properties are quite common in empirical finance. They are usually modelled by heteroscedastic processes, of which GARCH-type models are the most widespread. The original GARCH model was introduced by Bollerslev (1986) and can be formulated in the following way:

$\varepsilon_{t}=\sigma_{t} Z_{t}$ 

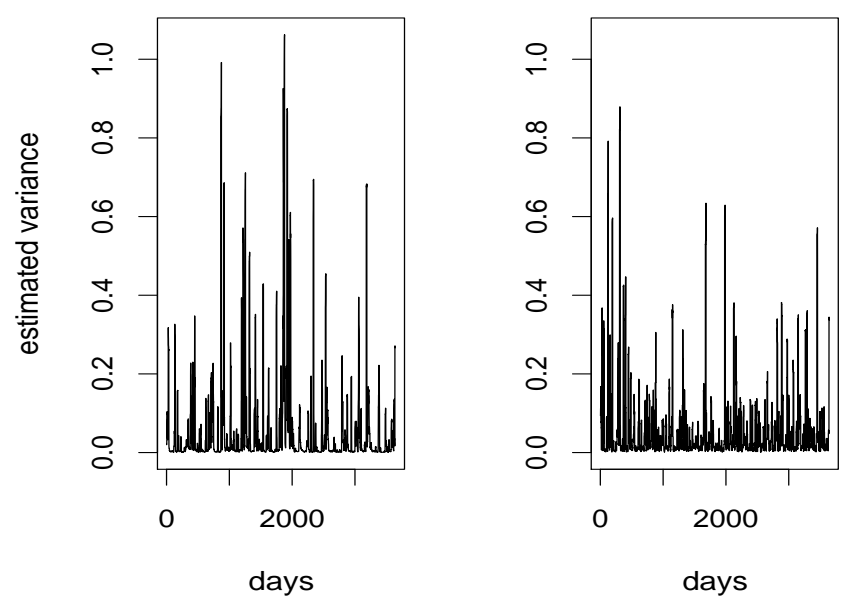

Fig. 7. A section of the estimated variance process of innovations (left) and of reshuffled innovations (right) at Vásárosnamény.

$\sigma_{t}^{2}=a_{0}+a_{1} \varepsilon_{t-1}^{2}+b_{1} \sigma_{t-1}^{2}$,

where $Z_{t}$-s are independent, zero-mean random variables with unit variance, and $\sigma_{t}^{2}$ is the time-varying conditional variance of $\varepsilon_{t} . a_{0}, a_{1}$ and $b_{1}$ are nonnegative parameters, which describe the dependence of $\sigma_{t}^{2}$ on the lagged value and on the lagged variance. (When $b_{1}=0$, we obtain the ARCH model introduced by Engle (1982).) Values of the $\varepsilon_{t}$ process are uncorrelated but interdependent through their squares. A comprehensive introduction into GARCH models is given e.g. in Hamilton (1994).

Because of its inherent heavy-tailedness (Mikosch and Starica, 2000), the above described original GARCH model is not directly suitable for river flow analysis. Instead, a more flexible heteroscedastic GARCH-type model can be used for modelling innovations, where the conditional variance is allowed to depend on the lagged values and on the lagged variance in a more complicated way ( $f$ is a given parametric bivariate function):

$\varepsilon_{t}=\sigma_{t} Z_{t}$

$\sigma_{t}^{2}=f\left(\varepsilon_{t-1}, \sigma_{t-1}^{2}\right)$.

Although Bühlmann and McNeil (2002) gives a general nonparametric method to identify $f$, we have not used this method because of computational difficulties. However, there is an easier - although not precise in the case of $b_{1} \neq 0$ - way to illustrate how $\sigma_{t}^{2}$ depends on $\varepsilon_{t-1}$. If the innovations are grouped (e.g. into 50 groups) according to their rank and the variance of the subsequent innovations are computed for all groups, we see that their conditional variance depends on the value of the previous innovation (cf. Fig. 8). (If the $\varepsilon_{t}$ process were independent, no pattern would appear.) According to Fig. 8, when the absolute value of the innovation is large, we expect the next innovation to have large absolute value as well. However, this empirical relation is far from the quadratic function obtained from the standard GARCH model.

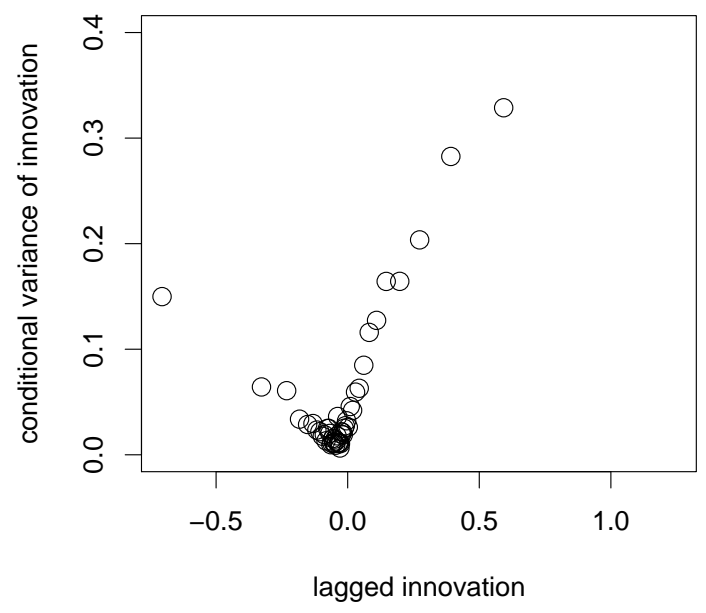

Fig. 8. Empirical conditional variance of innovations as a function of the previous value at Vásárosnamény.

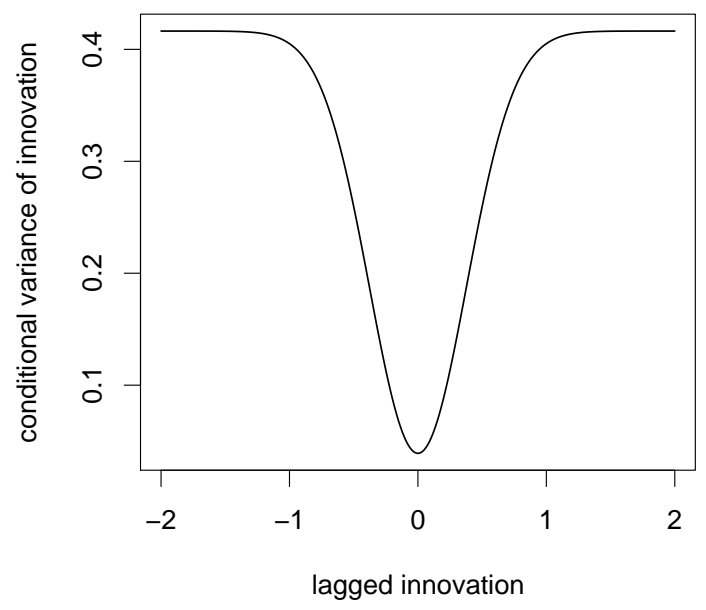

Fig. 9. Conditional variance of innovations from the fitted theoretical model.

Based on Fig. 8, we have specified $f$ (and thus the model) in the following form:

$\varepsilon_{t}=\sigma_{t} Z_{t}$

$\sigma_{t}^{2}=a_{0}+a_{1}\left(1-\exp \left(-s \varepsilon_{t-1}^{2}\right)\right)+b_{1} \sigma_{t-1}^{2}$.

How is this model working? When the absolute value of $\varepsilon_{t-1}$ is large, $\varepsilon_{t-1}$ has no incremental effect on the conditional variance. In this case $\sigma_{t}^{2}$ can be viewed as an autoregressive process:

$\sigma_{t}^{2} \approx a_{0}+a_{1}+b_{1} \sigma_{t-1}^{2}$.

When $\varepsilon_{t-1}$ is closer to zero, the process is similar to a GARCH-process:

$\sigma_{t}^{2} \approx a_{0}+a_{1} s \varepsilon_{t-1}^{2}+b_{1} \sigma_{t-1}^{2}$,

which in the neighbourhood of zero reduces to

$\sigma_{t}^{2} \approx a_{0}+b_{1} \sigma_{t-1}^{2}$. 

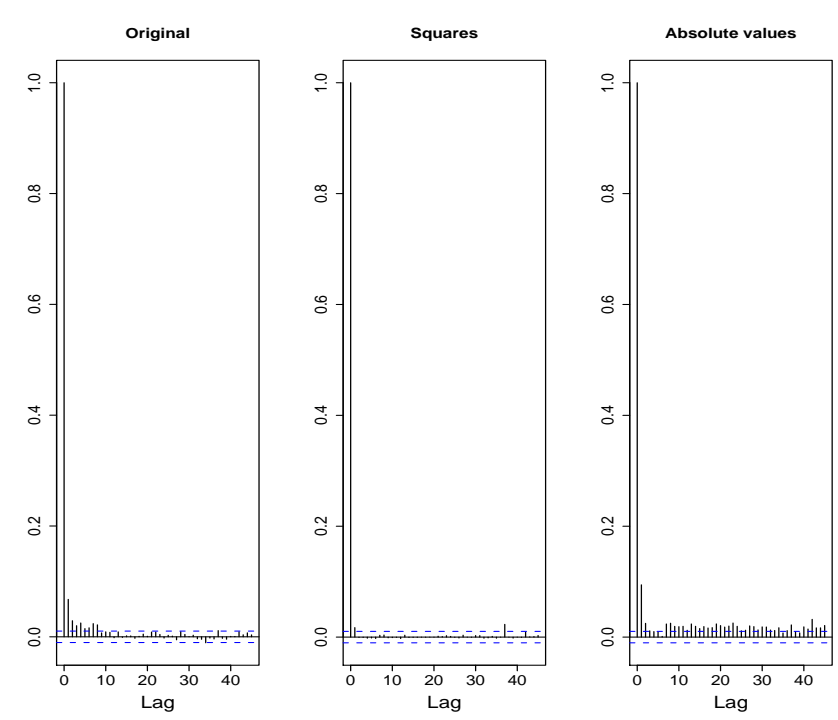

Fig. 10. Autocorrelation function of the original, squared and absolute valued GARCH-residual process at Vásárosnamény station.

As the effect of $\varepsilon_{t-1}$ changes smoothly between these extreme cases, the model may be called a smooth transition GARCH-type process. Put another way, there are basically two distinct regimes in the variance of the innovation process, and they can transform into one another in a smooth way. The theoretical smooth relationship between the conditional variance and the lagged innovations is displayed in Fig. 9.

After the conditional distribution (i.e. the distribution of $Z_{t}$ ) is specified, parameter estimation can be carried out by the method of conditional maximum likelihood (Hamilton, 1994). In the case of conditional normality, this method essentially maximises the following function:

$L\left(a_{0}, a_{1}, b_{1}, s\right)=\prod_{t=1}^{n} \frac{1}{\sqrt{2 \pi \sigma_{t}^{2}}} \exp \left(-\frac{\varepsilon_{t}^{2}}{2 \sigma_{t}^{2}}\right)$

where $\sigma_{t}^{2}$ is the conditional variance specified in Eq. (12). For the innovations of river discharge at Vásárosnamény the following model was obtained:

$\varepsilon_{t}=\sigma_{t} Z_{t}$

$\sigma_{t}^{2}=0.0062+0.38\left(1-\exp \left(-3.51 \varepsilon_{t-1}^{2}\right)\right)+0.46 \sigma_{t-1}^{2}$.

\section{Simulations from the FARIMA-GARCH model}

The $Z_{t}$ series in Eq. (11) (which we call GARCH-residuals) can be calculated recursively using the expression (14) for $\sigma_{t}^{2}$. As Fig. 10 shows, the squared and absolute valued residual process are no longer autocorrelated, although some negligible autocorrelation appears in the residual series itself. So the GARCH-residuals are much closer to independence than the pure innovation process, making simulation by bootstrapping more acceptable. As the distribution is more peaked and
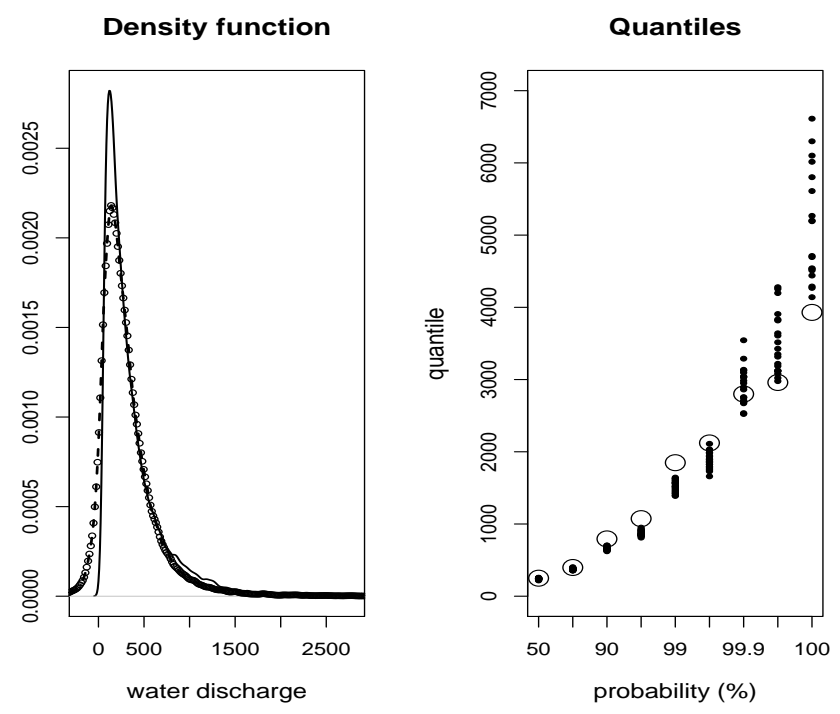

Fig. 11. Probability density and high quantiles (50, 70, 90, 95, 98, $99,99.5,99.95 \%$ and maximum) of observed (continuous/circled) and simulated (dotted) water discharge series at Vásárosnamény.

heavier-tailed than the standard normal one, the bootstrap procedure is indeed needed again. The shape of the distribution could also cause problems during estimation, however, it is quite common in the finance literature to use Gaussian conditional maximum likelihood even when the distribution is non-Gaussian (see e.g. McNeil and Frey (2000).)

Having simulated the GARCH-residuals, the $\sigma_{t}^{2}$ conditional variances and the $\varepsilon_{t}$ innovations are easily generated recursively from Eq. (14). After that, as earlier, the fractional ARIMA filter and the seasonal component can be applied to get synthetic streamflow series.

Figure 11 shows that the peakedness of the probability density is much better approximated by these simulations than by the simulations from the linear model. The 100-year maximum (which is quite important for flood risk estimation) is overestimated while quantiles in the range $90 \%-95 \%$ are slightly underestimated. The fit may improve when the generalised Pareto-distribution is used at the tails, or when the form of the model is more carefully chosen, e.g. by the nonparametric fitting procedure proposed by Bühlmann and McNeil (2002).

\section{Conclusions}

An overview of earlier results on statistical analysis of river flows is given in Lawrance and Kottegada (1977). Noakes et al. (1988) compared ARMA, fractional ARIMA and Markov-type models in terms of their one-year ahead forecasting ability. Periodic autoregressive moving average models were applied by Vecchia and Ballerini (1991) to describe the autocorrelation structure of monthly data. The role of long range dependence was studied by Ray (1993) and Beran (1994) with the findings that its importance lays in confidence bounds and uncertainty estimations rather than point 
forecasting. Seasonal long memory models were analysed recently in Montanari et al. (2000) and Ooms and Franses (2001) where further references can be found.

Our analysis proved that taking into account linear dependencies only, no matter at what length or precision, does not result in satisfactory description of the discharge series. This is particularly the case when extremes or high quantiles are concerned. From the numerous possible nonlinear models, a GARCH-type one was selected on the basis of autocorrelations of squares and absolute values and in view of the heteroscedasticity of the innovation process. Heuristic arguments for GARCH-innovations - the greater the previous innovation, the larger the variance of the next - may also be given in various possible ways. Were a rigorous connection between the innovations and the flood-generating weather patterns established, one could say: a high-value innovation indicates extreme weather patterns with unstable conditions, leading to high uncertainty in the next step. However, being a guess rather than an argument, we avoid to elaborate on this any further.

The original GARCH-philosophy prescribes the variance conditional on lagged values as a quadratic function. This property is meant to capture investor behaviour in financial markets where incoming shocking news cause greater uncertainty (the above mentioned quadratically increasing variance) for the next couple of days. Natural phenomena lack this psychological effect, therefore in our case there must be a bound for the increase of variance. This is what our modified GARCH-model intends to describe.

There is another way to look at our model. It captures the switch between high and low variance in an easily estimable way, i.e. by making use of the apparent statistical relationship between conditional variance and lagged innovations. Whether other processes like regime switching ones may be useful in modelling heteroscedasticity, or, in the GARCHtype context, what other variables the variance may depend on, are topics of further research.

Acknowledgements. This research was funded by Hungarian National Research and Development Project No. 3/067/2001 (project title: Establishing the Engineering and Scientific Bases of Flood Risk Assessment) and partially by National Scientific Research Fund OTKA, grant No. T 032725.

Edited by: T. Glade

Reviewed by: two referees

\section{References}

Beran, J.: A goodness of fit test for time series with long-range dependence, J. Roy. Statist. Soc., Series B, 54, 749-760, 1992.

Beran, J.: Statistics for long-memory processes, Chapman and Hall, New York, 1-315, 1994.

Bollerslev, T.: Generalised autoregressive conditional heteroscedasticity, J. of Econometrics, 31, 307-327, 1986.

Bühlmann, P. and McNeil, A.: An algorithm for nonparametric GARCH modelling, J. of Comput. Statist. and Data Analysis, 40, 665-683, 2002.

Cleveland, B. R., Cleveland, W. S., McRae, J. E., and Terpenning, I.: STL: A seasonal trend-decomposition procedure based on loess, J. of Off. Statist., 6, 3-73, 1990.

Engle, R. F.: Autoregressive Conditional Heteroskedasticity with Estimates of the Variance of the United Kingdom Inflation, Econometrica, 50, 987-1007, 1982.

Giraitis, L. and Surgailis, D.: A central limit theorem for quadratic forms in strongly dependent linear variables and applicationto asymptotical normality of Whittle's estimate, Prob. Th. Rel. Fields, 86, 87-104, 1990.

Giraitis, L. and Taqqu, M. S.: Whittle estimator for finite-variance non-Gaussian time series with long memory, Ann. Statist., 27, 178-203, 1999.

Hamilton, J. D.: Time series analysis, Princeton University Press, Princeton, N.J., 657-676, 1994.

Hurst, H. E.: Long-term storage capacity of reservoirs, Trans. of the Amer. Soc. of Civil Engineers, 770-808, 1951.

Lawrance, A. J. and Kottegada, N. T.: Stochastic modelling of river flow time series, J. Roy. Statist. Soc., Series A, 140, 1-31, 1977.

McNeil, A. and Frey, R.: Estimation of tail-related risk measures for heteroscedastic financial time series: an extreme value approach, J. of Empirical Finance, 7, 271-300, 2000.

Mikosch, T. and Starica, C.: Limit theory for the sample autocorrelations and extremes of a $\operatorname{GARCH}(1,1)$ process, Ann. Statist., 28, 1427-1451, 2000.

Montanari, A., Rosso, R., and Taqqu, M. S.: Fractionally differenced ARIMA models applied to hydrologic time series: Identification, estimation and simulation, Water Resources Research, 33, 1035-1044, 1997.

Montanari, A., Rosso, R., and Taqqu, M. S.: A seasonal fractionally differenced ARIMA model: an application to the Nile River monthly flows at Aswan, Water Resources Research, 36, 12491259, 2000.

Noakes, D. J., Hipel, K. W., McLeod, A. I., Jimenez, C., and Yakovitz, S.: Forecasting annual geophysical time series, Intern. J. of Forecasting, 1, 179-190, 1988.

Ooms, M. and Franses, P. H.: A seasonal periodic long memory model for monthly river flows, Environmental Modelling and Software, 16, 559-569, 2001.

Ray, B.: Modeling long-memory processes for optimal long-range prediction, J. of Time Series Analysis, 14, 511-525, 1993.

Vecchia, A. V. and Ballerini, R.: Testing for periodic autocorrelations in seasonal time series data, Biometrika, 78, 53-63, 1991 